\title{
Extrato Hidroetanólico de Gengibre (Zingiber officinale) Inibe a Oxidação da Lipoproteína de Baixa Densidade (LDL), In Vitro
}

\author{
Stêfani de Moraes ${ }^{1}$, Amanda Felipe Portella ${ }^{1}$, Andressa Leal Zambra ${ }^{1}$, Bruna Morgan da Silva ${ }^{1}$, \\ Natacha Cossettin Mori ${ }^{1}$, Isadora Aguirre Rosa ${ }^{2}$, Viviane Cecília Kessler Nunes Deuschle ${ }^{1}$, \\ Josiane Woutheres Bortolotto ${ }^{1}$, Gabriela Bonfanti Azzolin ${ }^{3}$
}

\begin{abstract}
RESUMO
Introdução: O uso de antioxidantes naturais para prevenir a ocorrência de aterosclerose e reduzir o risco de doenças cardiovasculares é uma tendência em ascensão. Objetivo: Considerando a oxidação da LDL como fator crucial no desenvolvimento da aterosclerose, e as propriedades antioxidantes, anti-inflamatórias e antiangiogênica do gengibre (Zingiber officinale), o objetivo do presente estudo é avaliar o conteúdo fitoquímico, mecanismos antioxidantes e o potencial do Extrato Hidroetanólico de Rizoma de Gengibre (EHEG) sobre a oxidação da lipoproteína de baixa densidade (LDL) de pacientes hipercolesterolêmicos, in vitro. Método: No EHEG foram avaliados teores de compostos fitoquímicos e mecanismos antioxidantes. A partir de amostras de soro dos pacientes ( $n=12)$, a fração LDL foi isolada, previamente tratada com EHEG (10 - $100 \mathrm{\mu g} / \mathrm{ml}$ ) e submetida à indução oxidativa com dicloridrato de 2,2'-Azobis (2-amidinopropano) (AAPH- $10 \mathrm{mM}$ ). Após, o nível de lipoperoxidação foi medido por meio da determinação espectrofotométrica de substâncias relativas ao ácido tiobarbitúrico (TBARS). Resultados: O EHEG possui compostos fenólicos totais $(357,68 \pm 39,80 \mathrm{mg} \mathrm{AG} / \mathrm{g})$, flavonoides $(30,37 \pm 5,10 \mathrm{mg} \mathrm{Q} / \mathrm{g})$, taninos $(51,99 \pm 11,88 \mathrm{mg} \mathrm{CA} / \mathrm{g})$ e vitamina $\mathrm{C}(21,53 \pm 1,43 \mathrm{mg}$ Vit $\mathrm{C} / \mathrm{g})$, além de apresentar atividade removedora de radicais de $\mathrm{NO}\left(\mathrm{IC}_{50}=40,53 \mu \mathrm{g} / \mathrm{mL}\right)$ e de $\mathrm{H}_{2} \mathrm{O}_{2}\left(\mathrm{IC}_{50}=1,42 \mathrm{mg} / \mathrm{mL}\right)$. Quanto à inibição da oxidação de LDL, observou-se que o EHEG, a partir da concentração de $50 \mu \mathrm{g} / \mathrm{ml}$, foi efetivo em reduzir o dano oxidativo causado pelo AAPH. Conclusão: O gengibre possui atividade protetora contra a formação de LDL oxidada, sugerindo que seus compostos fitoquímicos conferem-lhe atividade antioxidante, podendo ser efetivo na prevenção e/ou tratamento de doenças cardiovasculares, especialmente as relacionadas ao processo de aterosclerose.
\end{abstract}

Palavras-chave: Antioxidante. Aterosclerose. Hipercolesterolemia. Produtos naturais. Estresse oxidativo.

GINGER HYDROETHANOLIC EXTRACT INHIBITS OXIDATION OF LOW DENSITY LIPOPROTEIN (LDL), IN VITRO

\section{ABSTRACT}

Introduction: The use of natural antioxidants to prevent atherosclerosis and reduce the risk of cardiovascular disease is a growing trend. Purpose: Considering LDL oxidation as a crucial factor in the development of atherosclerosis, and the antioxidant, anti-inflammatory and anti-angiogenic properties of ginger (Zingiber officinale), the aim of the present study is to evaluate the phytochemical content, antioxidant mechanisms and the potential of Ginger Rhizome Hydroethanolic Extract (EHEG), on low density lipoprotein (LDL) oxidation of hypercholesterolemic patients, in vitro. Method: In EHEG were evaluated contents of phytochemical compounds and antioxidant mechanisms. From patient serum samples $(n=12)$, the LDL fraction was isolated, previously treated with EHEG $(10-100 \mu \mathrm{g} / \mathrm{ml})$ and subjected to oxidative induction with 2,2'-Azobis(2-methylpropionamidine) dihydrochloride (AAPH- 10 mM). Afterwards, the lipoperoxidation level was measured through spectrophotometric determination of thiobarbituric acid-related substances (TBARS). Results: EHEG has total phenolic compounds (357.68 $\pm 39.80 \mathrm{mg}$ $\mathrm{AG} / \mathrm{g})$, flavonoids $(30.37 \pm 5.10 \mathrm{mg} \mathrm{Q} / \mathrm{g})$, tannins $(51.99 \pm 11.88 \mathrm{mg} \mathrm{CA} / \mathrm{g})$ and vitamin C $(21.53 \pm 1.43 \mathrm{mg} \mathrm{Vit} \mathrm{C} / \mathrm{g})$, besides presents NO (IC50 = $40.53 \mathrm{\mu g} / \mathrm{mL})$ and $\mathrm{H}_{2} \mathrm{O}_{2}(\mathrm{IC} 50=1.42 \mathrm{mg} / \mathrm{mL})$ radical scavenging activity. Regarding inhibition of LDL oxidation, it was observed that the EHEG, from the concentration of $50 \mathrm{\mu g} / \mathrm{ml}$ was effective in reducing the oxidative damage caused by AAPH. Conclusion: Ginger has protective activity against the formation of oxidized LDL, suggesting that its phytochemical compounds confer antioxidant activity, and may be effective in the prevention and/or treatment of cardiovascular diseases, especially those related to the proce

Keywords: Antioxidant. Atherosclerosis. Hypercholesterolemia. Natural products. Oxidative stress.

RECEBIDO EM: 27/9/2019

MODIFICAÇÕES SOLICITADAS EM: 11/11/2019

ACEITO EM: 6/2/2020

\footnotetext{
${ }^{1}$ Universidade de Cruz Alta - Unicruz. Cruz Alta/RS, Brasil.

Universidade Federal de Santa Maria - UFSM. Santa Maria/RS, Brasil.

${ }^{3}$ Autora correspondente. Universidade de Cruz Alta - Unicruz. Rodovia Municipal Jacob Della Mea, s/n, km 5,6 - Parada Benito, Cruz Alta/RS, Brasil. CEP 98020-290. http://lattes.cnpq.br/1516086893514285. https://orcid.org/0000-0003-2602-6092. gbonfanti@unicruz.edu.br
} 


\section{INTRODUÇÃO}

As doenças cardiovasculares (DCVs) são a principal causa de morbidade e mortalidade em populações ocidentais (NETO et al., 2016), e, no Brasil, são a causa mais prevalente de morte dentre as doenças crônicas não transmissíveis (MALTA et al., 2019). As DCVs promovem altos custos que implicam impactos socioeconômicos importantes, divididos entre pacientes, familiares e o Estado, em razão da elevada frequência de internações hospitalares, carência de profissionais e aposentadoria antecipada (NETO et al., 2016).

O aumento do colesterol plasmático total é um importante fator de risco para a evolução das DCVs (MCCORMACK; DENT; BLAGDEN, 2016), posto que os efeitos patológicos da hipercolesterolemia devem-se especialmente à elevação dos níveis das lipoproteínas de baixa-densidade (LDL) na circulação (YAO et al., 2017). Essas, por sua vez, podem acumular-se no espaço subendotelial, quando estarão expostas aos produtos oxidativos das células endoteliais, macrófagos e células musculares lisas, que provocam o processo de lipoperoxidação, gerando, assim, a forma oxidada da LDL (LDL-ox), crucial na patogênese da aterosclerose. A LDL-ox induz as células a produzirem potentes ativadores celulares, promovendo a migração de células para o espaço subendotelial, que iniciam o processo de fagocitose das partículas de LDL-ox, formando as células espumosas. Posteriormente, há a ativação de uma cascata inflamatória e de coagulação, que dá origem à placa aterosclerótica. A placa tem um núcleo de material lipídico necrótico envolto por uma capa fibrosa suscetível a rupturas, as quais são responsáveis por ocasionar trombos, infarto do miocárdio, acidente vascular cerebral e até morte súbita (CARVAJAL, 2015; CHERNYAVSKIY et al., 2016).

A busca por estratégias eficazes usando antioxidantes naturais para prevenir a oxidação da LDL e reduzir o risco de DCVs, é uma tendência em ascensão. Investigações científicas demonstram o possível papel dos antioxidantes naturais, encontrados abundantemente em plantas, o reforço do mecanismo antioxidante endógeno e, ainda, o atraso ou supressão do estresse oxidativo envolvido no processo de aterogênese (ASSIS et al., 2017; SRINIVASAN, 2014).

Nesse contexto, o gengibre (Zingiber officinale) é uma planta perene, endêmica da Índia e cultivada no Sul e Sudeste da Ásia, África, América Latina e Austrália (KHODAIE; SADEGHPOOR, 2015), sendo amplamente utilizada como tempero e alimento, bem como agente terapêutico na medicina tradicional de diver- sas populações (AHMAD et al., 2015). No Brasil é encontrada em regiões quentes e úmidas, desde o Amazonas até o Paraná (CORREA JUNIOR, 1994).

Preparações fitoquímicas dessa espécie vegetal vêm mostrando-se efetivas na ação antioxidante, antiangiogênica (TABIBI et al., 2016), anti-inflamatória, anti-hiperglicêmica (SHIRISH et al., 2016), antitrombótica, imunomoduladora (AHMAD et al., 2015), analgésica e antibacteriana (KHODAIE; SADEGHPOOR, 2015), além de atuar em distúrbios gastrointestinais e renais (YANG et al., 2014).

Considerando, portanto, a LDL-ox um fator importante no processo patogênico da aterosclerose e consequentes DCVs, é de grande valor científico o estudo de compostos que possam atenuar esse processo e serem propostos como formas de tratamento e prevenção. Dessa forma, conhecendo a ampla utilização do gengibre e suas atividades antioxidantes, anti-inflamatórias e antiangiogênica já descritas, o objetivo do presente estudo é avaliar o conteúdo fitoquímico, mecanismos antioxidantes e o potencial do Extrato Hidroetanólico de Rizoma de Gengibre (Zingiber officinale) (EHEG) sobre a oxidação da lipoproteína de baixa densidade (LDL) de pacientes hipercolesterolêmicos, in vitro.

\section{METODOLOGIA}

\section{Amostra}

Foram selecionados 12 pacientes hipercolesterolêmicos que não faziam uso de medicamentos de forma contínua durante a coleta sanguínea para a realização de seus exames de rotina no Laboratório Escola de Análises Clínicas da Unicruz. Os dados gerais e de perfil lipídico dos pacientes encontram-se apresentados na Tabela 1. O presente estudo foi aprovado pelo Comitê de Ética em Pesquisa da Unicruz sob parecer número 991.132.

Tabela 1 - Caracterização dos indivíduos incluídos no estudo

\begin{tabular}{lc}
\hline \multicolumn{1}{c}{ Variável } & Dados \\
Sexo (F/M) & $9 / 3$ \\
Idade (anos) & $55,5 \pm 21,16$ \\
Colesterol total (mg/dL) & $217,58 \pm 18,93$ \\
Triglicerídeos (mg/dL) & $110,00 \pm 22,83$ \\
Colesterol HDL (mg/dL) & $68,57 \pm 21,47$ \\
Colesterol LDL (mg/dL) & $114,85 \pm 28,56$ \\
\hline
\end{tabular}

Fonte: Os autores. 


\section{Material vegetal}

O material vegetal (rizomas) foi obtido de fontes comerciais, devidamente identificado na Universidade de Cruz Alta (Unicruz) e utilizado imediatamente. As amostras de rizoma foram secas em estufa com circulação de ar $( \pm 40 \circ \mathrm{C})$, trituradas em moinho de facas e submetidas à maceração. Foi realizada a maceração hidroetanólica (EtOH: $\mathrm{H}_{2} \mathrm{O} 3: 2, \mathrm{v} / \mathrm{v}$ ) do material e o macerado foi submetido a agitações manuais diárias, por um período de sete dias (primeira maceração). Ao fim desse período, o material foi filtrado em algodão, seguindo-se de concentração em evaporador rotatório, à temperatura inferior a $40 \circ \mathrm{C}$, para eliminação do etanol. O material vegetal foi novamente recoberto com nova quantidade do mesmo solvente (segunda maceração) por mais sete dias. Este segundo líquido extrativo também foi filtrado, concentrado e reunido ao primeiro, obtendo-se, assim, o extrato hidroetanólico do rizoma de gengibre (EHEG) (SIMÕES et al., 2016). Esse foi seco até evaporação do líquido para obtenção do extrato na forma de pó.

\section{Quantificação espectrofotométrica de compostos fitoquímicos}

O conteúdo total de compostos fenólicos foi determinado com o reagente de Folin-Ciocalteu em meio alcalino, utilizando-se, para isto, a curva analítica com ácido gálico e a leitura em comprimento de onda de $725 \mathrm{~nm}$. As análises foram realizadas em triplicata. Os resultados foram expressos em miligramas de ácido gálico por grama de extrato em pó (mg AG/g) (SUBRAMANIAN; PADMANABAN; SARMA, 1965).

$O$ teor de flavonoides totais foi medido com base no método de cloreto de alumínio colorimétrico. A análise foi realizada em triplicata, as absorbâncias determinadas em $510 \mathrm{~nm}$ e os resultados expressos em miligramas de quercetina por grama de extrato em pó (mg Q/g) (ZHISHEN; MENGCHENG; JIANMING, 1999).

A quantificação de taninos condensados foi realizada a partir de uma modificação do método de Morrison et al. (1995). As análises foram realizadas em triplicata e as absorbâncias foram determinadas a $500 \mathrm{~nm}$. O teor de taninos foi expresso em miligramas de catequina por grama de extrato em pó (mg C/g), baseado na curva padrão de catequina.

$\mathrm{O}$ teor de vitamina $\mathrm{C}$ foi determinado utilizando o método descrito por Benderitter et al. (1998), com base na reação com 4-dinitrofenilhidrazina (DNPH), e calculado com base na curva padrão. As análises fo- ram realizadas em triplicata, determinadas a 520nm e os resultados expressos como miligramas de vitamina C por grama de extrato em pó (mg de VIT C/g).

\section{Determinação de compostos fenólicos por Clae}

A análise foi realizada em triplicata por meio da cromatografia líquida de alta eficiência (Clae), com coluna de fase reversa (C18). O método cromatográfico foi, de acordo com Evaristo e Leitão (2001), com algumas modificações. Foi utilizada fase móvel composta por ácido acético $1 \%(\mathrm{v} / \mathrm{v})$ em água $(\mathrm{A})$ e metanol (B), de acordo com um gradiente de eluição, volume de injeção de $5 \mu \mathrm{L}$, temperatura da coluna de $40^{\circ} \mathrm{C}$ e vazão de $1,2 \mathrm{~mL} / \mathrm{min}$. $O$ tempo da corrida foi de $45 \mathrm{~min}$ e a detecção foi realizada em $280 \mathrm{~nm}$. O extrato foi avaliado para a presença dos seguintes compostos fenólicos: rutina, quercetina, ácido clorogênico, (-)-epicatequina, ácido gálico, apigenina, ácido elágico e ácido rosmarínico. A identificação foi feita utilizando os cromatogramas obtidos em cada injeção, comparado-os com o espectro de absorção e tempo de retenção dos padrões.

\section{Avaliação dos mecanismos antioxidantes}

O efeito de remoção do EHEG sobre o radical óxido nítrico (NO) foi medido de acordo com o método de Sreejayan e Rao (1997). Para o ensaio, o nitroprussiato de sódio (10 $\mathrm{mM}$ ) foi misturado com extrato em diferentes concentrações, incubado por $150 \mathrm{~min}$ e depois misturado com $0,5 \mathrm{~mL}$ de reagente de Griess, seguido de medida de absorbância a $546 \mathrm{~nm}$. No controle negativo, o extrato foi substituído por PBS (tampão fosfato salina). A capacidade de remoção de NO foi calculada usando a seguinte equação: Scavenger de NO $(\%)=[1$ - (Absorbância do extrato/Absorbância do controle)] $\times 100$. A expressão dos resultados foi dada pela IC50 (em $\mu \mathrm{g} / \mathrm{mL})$, calculada com auxílio da equação da reta interpolada com os dados de concentração (eixo das abcissas) e Scavenger de NO (\%) (eixo das ordenadas). Os resultados obtidos foram comparados com o padrão de ácido gálico.

A habilidade do EHEG de remover peróxido de hidrogênio $\left(\mathrm{H}_{2} \mathrm{O}_{2}\right)$ foi determinada por meio do acompanhamento da taxa de decomposição do $\mathrm{H}_{2} \mathrm{O}_{2} \mathrm{em}$ $230 \mathrm{~nm}$, quando incubado com diferentes concentrações do extrato, conforme Ruch, Cheng e Klaunig (1989). A capacidade de remoção foi calculada usando a seguinte equação: Scavenger de $\mathrm{H}_{2} \mathrm{O}_{2}(\%)=[($ Absorbância do controle - Absorbância do teste)/Absorbância do controle] $x$ 100. A expressão dos resultados foi dada pela IC50 (em $\mu \mathrm{g} / \mathrm{mL})$, calculada com auxí- 
lio da equação da reta interpolada com os dados de concentração (eixo das abscissas) e Scavenger de $\mathrm{H}_{2} \mathrm{O}_{2}$ (\%) (eixo das ordenadas). Os resultados obtidos foram comparados com o padrão de ácido gálico.

\section{Ensaio de oxidação de LDL}

As amostras de sangue foram coletadas sem anticoagulante e, após, centrifugadas por 10 minutos a 3.000 RPMs para a obtenção do soro. Para o isolamento da LDL, a cada $100 \mu \mathrm{L}$ de soro foi adicionado 1 $\mathrm{ml}$ de tampão citrato de sódio $(0,064 \mathrm{M})$ e heparina (50.000 UI/L) em pH 5,04. Após centrifugação a 3.000 RPM por 10 minutos, as lipoproteínas insolúveis foram precipitadas e posteriormente diluídas em $1 \mathrm{~mL}$ de PBS, sendo o sobrenadante descartado (WIELAND; SEIDEL, 1983). O conteúdo de proteínas presente na amostra, então, foi medido pelo método de Lowry modificado (PETERSON, 1977).

As lipoproteínas ajustadas a $100 \mu \mathrm{g} / \mathrm{mL}$ foram pré-incubadas com o EHEG nas concentrações de 10 - $100 \mu \mathrm{g} / \mathrm{mL}$, por $30 \mathrm{~min}$. Após os pré-tratamentos, a modificação oxidativa da LDL deu-se por incubação com $10 \mathrm{mM}$ de dicloridrato de 2,2'-Azobis (2-amidinopropano) (AAPH), por 2 horas a $37{ }^{\circ} \mathrm{C}$. Para fins de comparação do efeito do EHEG com um composto fitoquímico de conhecida atividade antioxidante, utilizou-se ácido gálico $(50 \mu \mathrm{g} / \mathrm{mL})$ como controle positivo.

Ao final do período de incubação foi avaliado o nível de peroxidação lipídica pela medida de Substâncias Reativas ao Ácido Tiobarbitúrico (TBARS) espectrofotometricamente, usando ácido Tiobarbitúrico 0,67\% (TBA) e ácido tricloroacético $20 \%$ (TCA). Os resultados foram expressos em nmol de malondialdeído (MDA)/mL (OKAWA; OHISHI; YAGI, 1979).

\section{Análise estatística}

Os resultados obtidos foram expressos por média \pm erro padrão da média (EPM). As diferenças foram avaliadas utilizando Anova de uma via seguida do pós-teste Tukey de comparações múltiplas. Os valores com $p \leq 0,05$ foram considerados significativamente diferentes.

\section{RESULTADOS E DISCUSSÃO}

O presente estudo demonstra a capacidade do extrato hidroetanólico do rizoma de gengibre (EHEG) de proteger partículas de LDL de pacientes hipercolesterolêmicos do processo de oxidação in vitro, sugerindo seu papel benéfico na prevenção e terapia da aterosclerose.
Analisando a Figura 1, podemos observar que o composto dicloridrato de 2,2'-azobis (2-amidinopropano) (AAPH) foi efetivo na concentração utilizada para a indução da lipoperoxidação, demonstrada por meio dos níveis elevados de malondialdeído (MDA), um dos produtos finais desse processo. O AAPH gera radicais peroxila que levam à oxidação de lipídeos a uma temperatura fisiológica ( $37^{\circ} \mathrm{C} \mathrm{Cm} \mathrm{pH}$ neutro) e a uma velocidade constante e reprodutível (PELUSO et al., 2002). Diante disso, o AAPH tem sido extensivamente utilizado como uma fonte previsível e controlável de radicais livres, mimetizando o estresse oxidativo fisiológico em estudos de oxidação de eritrócitos, plasma, sangue total, células, tecidos e, até mesmo, de forma sistêmica (HE et al., 2003), e em injúrias oxidativas que ocorrem em lipídeos e proteínas de LDL durante o processo aterogênico (BARCELOS et al., 2011).

Quanto ao efeito do EHEG, podemos observar que o mesmo se mostrou eficaz em reduzir a oxidação das LDLs a partir da concentração de $50 \mu \mathrm{g} / \mathrm{ml}$ (Figura 1 ), tornando os níveis de MDA nesses grupos semeIhantes aos do grupo controle não induzido, e sugerindo uma completa proteção dessas partículas. Além disso, pode-se observar que o EHEG preveniu a oxidação das LDLs de forma semelhante ao ácido gálico, um antioxidante bem-estabelecido e fundamentado na literatura (NAKSURIYA; OKONOGI, 2015), que foi utilizado no presente trabalho como controle positivo de ação antioxidante. Existem vários ensaios para a avaliação da oxidação de LDL. Dentre eles, o TBARS quantifica espectrofotometricamente o nível de MDA, que é um dos produtos finais da reação em cadeia da lipoperoxidação (OKAWA; OHISHI; YAGI, 1979) e, portanto, demonstra o nível de oxidação da LDL ao final do período de incubação. Dessa forma, esse ensaio tem sido utilizado em outros estudos que avaliam a capacidade de produtos naturais de inibir a oxidação de LDL (DOS SANTOS et al., 2018; CAl; CHEN; JIANG, 2018).

O papel do estresse oxidativo na formação de LDL-ox e seu envolvimento no processo de aterogênese já é amplamente discutido na literatura (CARVAJAL, 2015; CHERNYAVSKIY et al., 2016). Assim, a busca de antioxidantes provenientes de fontes naturais que possam retardar ou inibir esse processo, é importante estratégia de promoção à saúde da população.

Nesse contexto, há um interesse renovado no gengibre por suas propriedades antioxidantes e cardioprotetoras que vêm sendo demonstradas. No estudo de Fuhrman et al. (2013) investigou-se o efeito in vivo do extrato de gengibre sobre o desenvolvimento de aterosclerose em camundongos com deficiência 
Figura 1 - Efeito protetor do extrato hidroetanólico do gengibre (EHEG) na oxidação da lipoproteína de baixa densidade (LDL)

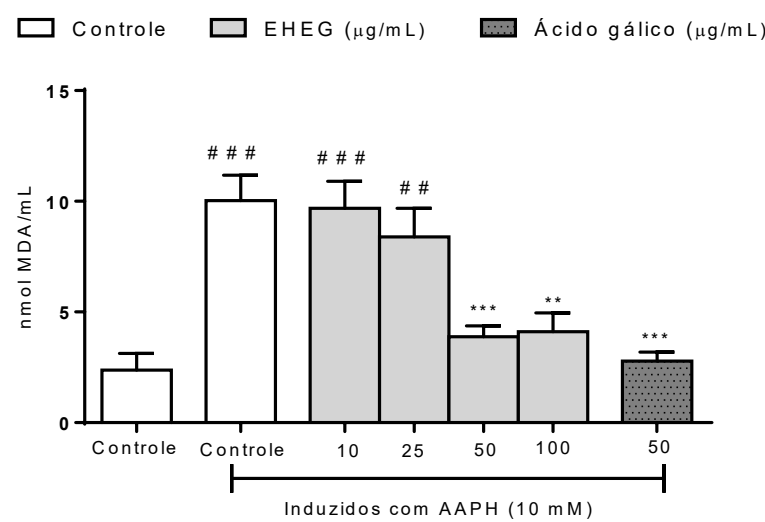

Efeito protetor do extrato hidroetanólico do gengibre (EHEG) na oxidação da lipoproteína de baixa densidade (LDL), verificado a partir dos níveis de malondialdeído (MDA). ${ }^{* * *} \mathrm{p}<0,001,{ }^{* *} \mathrm{p}<0,01$, diferentes do controle

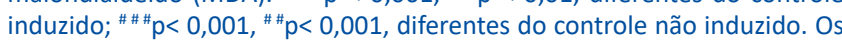
dados foram verificados por análise de variância (Anova) seguida de teste de Tukey, e expressos como média \pm erro padrão da média $(n=12)$.

$$
\text { Fonte: Os autores. }
$$

de Apolipoproteína E, chegando ao resultado de que o consumo de diferentes concentrações de extrato de gengibre reduzia em até $60 \%$ a oxidação do colesterol LDL. Já outro estudo acerca das atividades antioxidantes do rizoma do gengibre, demonstrou a capacidade de diferentes extratos de impedir a oxidação de LDL induzida por íon $\mathrm{Cu}^{2+} \mathrm{e}$ atribuiu tal efeito à presença de compostos bioativos fenólicos encontrados no gengibre, tais como o 6-gingerols, 8-gingerols, 10-gingerols e 6-shogaol (GUNATHILAKE; RUPASINGHE, 2014). Esse, entretanto, é o primeiro estudo que demonstra tais resultados com um extrato hidroetanólico e em modelo de indução com AAPH, que mimetiza o processo de oxidação fisiológica.

De fato, o gengibre é conhecido como um recurso natural de ampla distribuição e baixo custo, com altos teores de compostos fitoquímicos, que conferem a ele pronunciado efeito antioxidante (AKINOLA; AHMAD; MAZIAH, 2014; GHASEMZADEH; JAAFAR; RAHMAT, 2010). No presente estudo foi possível quantificar teores de compostos fenólicos totais, flavonoides, taninos condensados e vitamina C (Tabela 1 ).

Dentre os compostos quantificados, destacam-se os flavonoides que possuem dois anéis aromáticos ligados por meio de três carbonos que formam um heterociclo oxigenado, e que apresentam propriedades antioxidantes à medida que inativam os radicais livres, convertendo-os em radicais livres relativamente inofensivos (VESKOUKIS; TSATSAKIS; KOURETAS, 2012). São encontrados em diversos alimentos do
Tabela 1 - Conteúdo de compostos fitoquímicos do Extrato Hidroetanólico de Gengibre (EHEG)

\begin{tabular}{lc}
\hline Compostos fenólicos totais (mg AG/g) & $357,68 \pm 39,80$ \\
Compostos flavonoides totais (mg Q/g) & $30,37 \pm 5,10$ \\
Taninos condensados (mg CA/g) & $51,99 \pm 11,88$ \\
Vitamina C (mg Vit C/g) & $21,53 \pm 1,43$ \\
\hline
\end{tabular}

Os resultados são expressos em média \pm EPM de três determinações. $A G=$ ácido gálico; $\mathrm{Q}=$ quercitina; $\mathrm{CA}=$ catequina, Vit $\mathrm{C}=$ vitamina $\mathrm{C}$.

Fonte: Os autores.

reino vegetal, e demonstram possuir propriedades antioxidantes, anti-inflamatórias e vasodilatadoras que contribuem significativamente para a saúde humana, reduzindo o risco de DCV (FERRERA et al., 2016). Estudos in vitro mostraram a grande capacidade dos flavonoides em inibir a oxidação da LDL, chegando a ser superior à de antioxidantes clássicos, como o alfa-tocoferol, sugerindo efeito antiaterogênico dos flavonoides (ABOURASHED, 2013; VINSON et al., 1995).

Já a Vitamina C (ácido ascórbico) é uma vitamina hidrossolúvel, sintetizada a partir da glicose por plantas e muitos animais, mas, em razão da ausência de maquinaria enzimática específica, não é sintetizada pelo homem. Existem hipóteses bem-fundamentas que a vitamina $C$ pode desempenhar um papel fundamental na prevenção de DCV, por suas capacidades antioxidantes e o papel desses antioxidantes na prevenção de alterações oxidativas da LDL (SALVAYRE; NEGRE-SALVAYRE; CAMARÉ, 2016).

$\mathrm{Na}$ análise dos compostos fenólicos por Clae, foi possível verificar a ausência dos compostos testados: rutina, quercetina, ácido clorogênico, (-)-epicatequina, ácido gálico, apigenina, ácido elágico e ácido rosmarínico (Figura 2), indicando que a atividade antioxidante evidenciada se deve aos outros compostos que foram quantificados pelo método espectrofotométrico deste estudo. Tal resultado está relacionado à particularidade de conteúdo fitoquímico da espécie, que já apresenta descrito na literatura os compostos 6-gingerols, 8-gingerols, 10-gingerols e 6-shogaol como principais compostos fenólicos (GUNATHILAKE; RUPASINGHE, 2014).

Com o intuito de investigar os mecanismos da ação antioxidante do EHEG, o presente estudo avaliou sua a capacidade removedora ante as espécies reativas peróxido de hidrogênio $\left(\mathrm{H}_{2} \mathrm{O}_{2}\right)$ e radical óxido nítrico (NO) (Tabela 2). As espécies reativas de oxigênio e nitrogênio, como o $\mathrm{H}_{2} \mathrm{O}_{2}$ e o óxido nítrico, conferem regulação redox de funções celulares essenciais, envolvidas na diferenciação, proliferação, migração e apoptose, além de iniciar e catalisar respostas de es- 
Figura 2 - Determinação de compostos fenólicos por Cromatografia Líquida de Alta eficiência

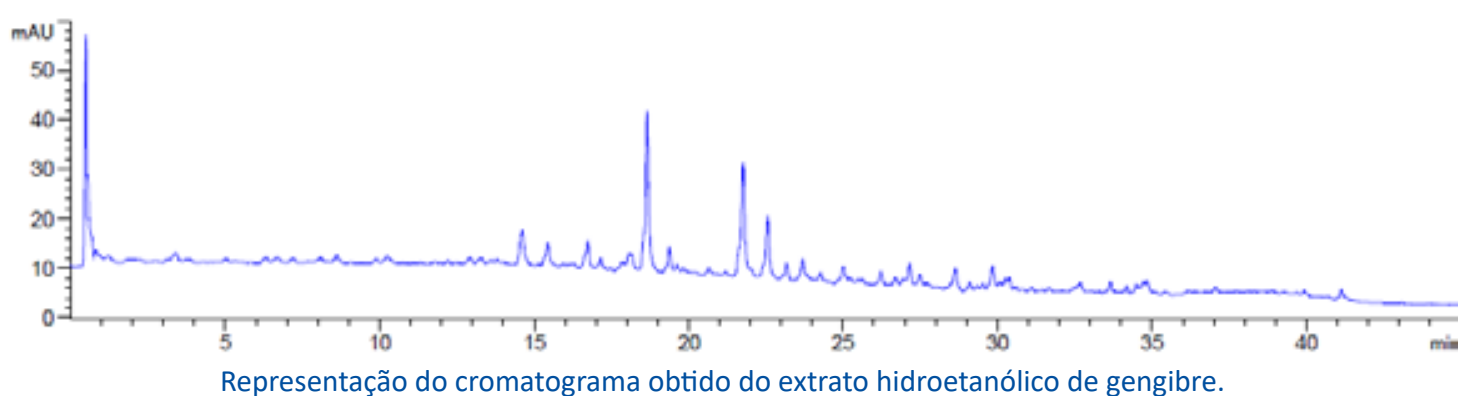

Fonte: Os autores.

Tabela 2 - Mecanismos antioxidantes avaliados no Extrato Hidroetanólico de Gengibre (EHEG) e controle positivo Ácido Gálico

\begin{tabular}{lcc}
\hline & EHEG & Ácido gálico \\
Capacidade removedora de $\mathrm{NO}(\mu \mathrm{g} / \mathrm{mL})$ & $\mathrm{IC} 50=40,53$ & $\mathrm{IC} 50=2,04$ \\
Capacidade removedora $\mathrm{H}_{2} \mathrm{O}_{2}(\mu \mathrm{g} / \mathrm{mL})$ & $\mathrm{IC} 50=1,42$ & $\mathrm{IC} 50=210,61$ \\
\hline
\end{tabular}

EHEG = extrato hidroetanólico de gengibre; $\mathrm{NO}$ = radical óxido nítrico; $\mathrm{H}_{2} \mathrm{O}_{2}=$ peróxido de hidrogênio.

Fonte: Os autores.

tresse adaptativo, entretanto, quando em excesso, podem atacar biomoléculas, causando danos e alterando sua funcionalidade, o que está relacionado com diversas patologias associadas ao estresse oxidativo, dentre elas a aterosclerose (DAIBER et al., 2017).

O NO é um radical livre considerado tóxico ou protetor, dependendo da sua concentração, localização e reação com as demais espécies reativas (TAIN; HSU, 2017). É descrito como um importante mediador do sistema imunológico na resposta inflamatória, e apresenta múltiplos efeitos biológicos, relacionados com a regulação da pressão arterial por meio do relaxamento do músculo liso e inibição da agregação plaquetária (CABALLANO-INFANTES et al., 2017). De maneira contraditória, entretanto, pode estar envolvido na produção de radicais citotóxicos, uma vez que pode reagir com o radical superóxido e produzir o ânion peroxinitrito e dióxido de nitrogênio, que iniciam a peroxidação lipídica e potencializam a lesão inflamatória (CERQUEIRA; YOSHIDA, 2002). Sabendo, portanto, que o EHEG aqui estudado demonstrou ação removedora de radicais NO, pode-se sugerir que esse mecanismo antioxidante esteja envolvido nas suas ações terapêuticas já descritas na literatura e exploradas pela medicina popular como ação anti-inflamatória, hepatoprotetora, antioxidante e de atenuação da peroxidação lipídica. Observa-se, no entanto, que a capacidade removedora desse radical é menor no extrato do que no ácido gálico isolado, utilizado como controle positivo. Considerando que no extrato não foi identificada a presença de ácido gálico, sugere-se que os compostos presentes no extrato, apesar de exercerem essa atividade antioxidante, o fazem com menor potência do que o controle positivo utilizado.

Da mesma forma, o EHEG também demonstrou capacidade removedora de $\mathrm{H}_{2} \mathrm{O}_{2}$, espécie reativa de oxigênio que, apesar de essencial em vias de sinalização que determinam a viabilidade celular e capacidade da célula de combater patógenos (SIES, 2017), em condições patológicas, como na hipercolesterolemia, seu nível aumentado leva à infiltração de células inflamatórias, oxidação lipídica e formação de células em espuma que contribuem significativamente para a inflamação vascular e o desenvolvimento da lesão aterosclerótica (BYON; HEATH; CHEN, 2016). Cabe ressaltar que o extrato apresentou capacidade tal removedora maior que o controle positivo utilizado, sugerindo que o material vegetal possui compostos fenólicos que podem exercer tal efeito antioxidante de forma aditiva ou sinérgica, apresentando-se como uma vantagem a compostos antioxidantes isolados.

\section{CONCLUSÃO}

O extrato hidroetanólico de gengibre possui propriedades antioxidantes, sendo capaz de proteger a LDL humana da oxidação e ser efetivo na prevenção e/ou terapia de doenças cardiovasculares decorrentes do processo de aterosclerose. Tal ação antioxidante pode ser mediada pela capacidade de remoção de radicais de óxido nítrico e peróxido de hidrogênio, demonstradas nesse estudo, dentre outros mecanismos. Ainda, é notável a presença de compostos fito- 
químicos com mecanismos antioxidantes que podem ter efeitos benéficos complementares sobre a saúde cardiovascular. Dessa forma, exalta-se o potencial do gengibre para o desenvolvimento de produtos fitoterápicos que atuem de forma complementar e integrativa, melhorando a saúde da população, especialmente no âmbito das doenças cardiovasculares.

\section{REFERÊNCIAS}

ABOURASHED, E. A. Bioavailability of plant-derived antioxidants. Antioxidants, v. 2, n. 4, p. 309-325, 2013.

AHMAD, B. et al. A review on pharmacological properties of zingerone (4-(4-Hydroxy-3-methoxyphenyl)-2-butanone). The Scientific World Journal, v. 2.015, p. 1-6, 2015.

AKINOLA, A. A.; AHMAD, S.; MAZIAH, M. Total anti-oxidant capacity, flavonoid, phenolic acid and polyphenol content in ten selected species of Zingiberaceae rhizomes. African Journal of Traditional, Complementary and Alternative Medicines, v. 11, n. 3, p. 7-13, 2014.

ASSIS, R. P. et al. Combined Effects of Curcumin and Lycopene or Bixin in Yoghurt on Inhibition of LDL Oxidation and Increases in HDL and Paraoxonase Levels in Streptozotocin-Diabetic Rats. International Journal of Molecular Sciences, v. 18, n. 4, p. 332. 2017.

BARCELOS, R. P. et al. Thiosemicarbazone derivate protects from AAPH and Cu 2+-induced LDL oxidation. Life Sciences, v. 89, n. 1, p. 20-28, 2011.

BENDERITTER, M. et al. Studies by electron paramagnetic resonance of the importance of iron in the hydroxyl scavenging properties of ascorbic acid in plasma: effects of iron chelators. Fundamental \& Clinical Pharmacology, v. 12, n. 5, p. 510-516, 1998.

BYON, C. H.; HEATH, J. M.; CHEN, Y. Redox signaling in cardiovascular pathophysiology: A focus on hydrogen peroxide and vascular smooth muscle cells. Redox Biology, v. 9, p. 244-253, 2016.

CABALLANO-INFANTES, E. et al. Regulation of mitochondrial function and endoplasmic reticulum stress by nitric oxide in pluripotent stem cells. World Journal of Stem Cells, v. 9, n. 2, p. 26-36, 2017.

CAI, R.; CHEN, S.; JIANG, S. Chlorogenic acid inhibits non-enzymatic glycation and oxidation of low density lipoprotein. Journal of Zhejiang University, Medical Sciences, v. 47, n. 1, p. 27-34, 2018.

CARVAJAL, C. C. LDL oxidada y la aterosclerosis. Medicina Legal de Costa Rica, v. 32, n. 1, p. 161-169, 2015.

CERQUEIRA, N. F.; YOSHIDA, W. B. Óxido Nítrico. Revisão. Acta Cirúrgica Brasileira, v. 17, n. 6, p. 417-423, 2002.

CHERNYAVSKIY, I. et al. Atherogenesis: hyperhomocysteinemia interactions with LDL, macrophage function, paraoxonase 1, and exercise. Annals of the New York Academy of Sciences, v. 1.363, n. 1, p. 138-154, 2016.

CORREA JUNIOR, C. Cultivo de plantas medicinais, condimentares e aromáticas. 2. ed. Jaboticabal: Funep, 1994.
DAIBER, A. et al. Taking up the cudgels for the traditional reactive oxygen and nitrogen species detection assays and their use in the cardiovascular system. Redox Biology, v. 12, p. 35-49, 2017.

DOS SANTOS, M. M. et al. Syzygium cumini leaf extract inhibits LDL oxidation, but does not protect the liproprotein from glycation. Journal of Ethnopharmacology, v. 210, p. 69-79, 2018.

EVARISTO, I. M.; LEITÃO, M. C. Identificação e quantificação por DAD-HPLC, da fraç̧ão fenólica contida em folhas de Quercus suber L. Silva Lusitana, v. 9, n. 2, p. 135-141, 2001.

FERRERA, T. S. et al. Substâncias fenólicas, flavonoides e capacidade antioxidante em erveiras sob diferentes coberturas do solo e sombreamentos. Revista Brasileira de Plantas Medicinais, v. 18, n. 2, suppl.1, p. 588-596, 2016.

FUHRMAN, B. et al. Ginger extract consumption reduces plasma cholesterol, inhibits LDL oxidation and attenuates development of atherosclerosis in atherosclerotic, apolipoprotein E-deficient mice. The Journal of Nutrition, v. 130, n. 5, p. 1.124-1.131, 2000.

GHASEMZADEH, A.; JAAFAR, H. Z.; RAHMAT, A. Identification and concentration of some flavonoid components in Malaysian young ginger (Zingiber officinale Roscoe) varieties by a high performance liquid chromatography method. Molecules, v. 15, n. 9, p. 6.231-6.243, 2010.

GUNATHILAKE, K. D. P.; RUPASINGHE, H. P. V. Inhibition of human low-density lipoprotein oxidation in vitro by ginger extracts. Journal of Medicinal Food, v. 17, n. 4, p. 424-431, 2014.

HE, R. et al. A new oxidative stress model, 2, 2-azobis (2-amidinopropane) dihydrochloride induces cardiovascular damages in chicken embryo. PloS One, v. 8, n. 3, 2003.

KHODAIE, L.; SADEGHPOOR, O. Ginger from ancient times to the new outlook. Jundishapur Journal of Natural Pharmaceutical Products, v. 10, n. 1, 2015.

MALTA, D. C. et al. Probabilidade de morte prematura por doenças crônicas não transmissíveis, Brasil e Regiões, projeções para 2025. Revista Brasileira de Epidemiologia, v. 22, 2019.

MCCORMACK, T.; DENT, R.; BLAGDEN, M. Very low LDL-C levels may safely provide additional clinical cardiovascular benefit: the evidence to date. International Journal of Clinical Practice, v. 70, n. 11, p. 886-887, 2016.

MORRISON, M. et al. Determination of Lignin and Tannin contents of cowpea seeds coats. Annals of Botany, v. 76, n. 1, p. 287-290, 1995.

NAKSURIYA, O.; OKONOGI, S. Comparison and combination effects on antioxidant power of curcumin with gallic acid, ascorbic acid, and xanthone. Drug discoveries \& therapeutics, v. 9, n. 2, p. 136-141, 2015.

NETO, J. R. F et al. Erica: prevalence of dyslipidemia in Brazilian adolescents. Revista de Saúde Pública, v. 50, p. 1-10, 2016.

OKAWA, H.; OHISHI, N.; YAGI, K. Assay for lipid peroxides in animal tissues by thiobarbituric acid reaction. Analytical Biochemistry, v. 95, n. 2, p. 351-358, 1979. 
PELUSO, I. et al. Intestinal motility disorder induced by free radicals: a new model mimicking oxidative stress in gut. Pharmacological Research, v. 46, n. 6, p. 533-538, 2002.

PETERSON, G. L. A simplification of the protein assay method of Lowry et al which is more generally applicable. Analytical Biochemistry, v. 83, n. 2, p. 346-356, 1977.

RUCH, R. J.; CHENG, S.; KLAUNIG, J. E. Prevention of cytotoxicity and inhibition of intercellular communication by antioxidant catechins isolated from Chinese green tea. Carcinogenesis, v. 10, n. 6, p. 1.003-1.008, 1989.

SALVAYRE, R.; NEGRE-SALVAYRE, A.; CAMARÉ, C. Oxidative theory of atherosclerosis and antioxidants. Biochimie, $\mathrm{V}$. 125, p. 281-296, 2016.

SHIRISH, D. et al. Zingiber officinale attenuates retinal microvascular changes in diabetic rats via anti-inflammatory and antiangiogenic mechanisms. MolecularVision, v. 22, p. 599-609, 2016.

SIES, H. Hydrogen peroxide as a central redox signaling molecule in physiological oxidative stress: Oxidative eustress. Redox Biology, v. 11, p. 613-619, 2017.

SIMÕES, C. M. O. et al. Farmacognosia: do produto natural ao medicamento. Porto Alegre: Artmed Editora, 2016.

SREEJAYAN, N.; RAO, M. N. A. Nitric oxide scavenging by curcuminoids. Journal of Pharmacy and Pharmacology, $v$. 49, p. 105-107, 1997.

SRINIVASAN, K. Antioxidant potential of spices and their active constituents. Critical Reviews in Food Science and Nutrition, v. 54, n. 3, p. 352-372, 2014.

SUBRAMANIAN, K. N.; PADMANABAN, G.; SARMA, P. S. Folin-Ciocalteu reagent for the estimation of siderochromes. Analitycal Biochemistry, v. 12, p. 106-112, 1965.

$\mathrm{TABIBI}, \mathrm{H}$. et al. Effects of ginger on serum lipids and lipoproteins in peritoneal dialysis patients: a randomized controlled trial. Peritoneal Dialysis International, v. 36, n. 2, p. 140-145, 2016.

TAIN, Y.; HSU, C. Interplay between Oxidative Stress and Nutrient Sensing Signaling in the Developmental Origins of Cardiovascular Disease. International Journal of Molecular Sciences, v. 18, n. 4, p. 841, 2017.

VESKOUKIS, A. S.; TSATSAKIS, A. M.; KOURETAS, D. Dietary oxidative stress and antioxidant defense with an emphasis on plant extract administration. Cell Stress and Chaperones, v. 17, n. 1, p. 11-21, 2012.

VINSON, J. A. et al. Plant polyphenols exhibit lipoprotein-bound antioxidant activity using an in vitro oxidation model for heart disease. Journal of agricultural and Food chemistry, v. 43, n. 11, p. 2.798-2.799, 1995.

WIELAND, H.; SEIDEL, D. A simple specific method for precipitation of low density lipoproteins. Journal of Lipid Research, v. 24, n. 7, p. 904-909, 1983.

YANG, M. et al. Ginger extract diminishes chronic fructose consumption-induced kidney injury through suppression of renal overexpression of proinflammatory cytokines in rats. BMC Complementary and Alternative Medicine, v. 14, $\mathrm{n}$. 174, p. 1-12, 2014.
YAO, Y. et al. Klotho ameliorates oxidized low density lipoprotein (ox-LDL) - induced oxidative stress via regulating LOX-1 and PI3K/Akt/eNOS pathways. Lipids in Health and Disease, v. 16, n. 1, p. 77, 2017.

ZHISHEN, J.; MENGCHENG, T.; JIANMING, W. The determination of flavonoid contents in mulberry and their scavenging effects on superoxide radicals. Food Chemistry, v. 64, n. 4, p. 555-559, 1999. 